\title{
PROGNOSTICO DE PACIENTES NEUROTICOS EM TRATAMENTO AMBULATORIAL
}

\author{
Laerte Moreira da Silva *: \\ LUCI MOREIRA DA SILva**
}

A maior parte dos estudos que visam determinar o prognóstico nas neuroses leva em consideração fatores individuais e ambientais de cada caso estudado. Para Fenichel ${ }^{3}$, as conclusões a que chegam os diversos autores ficam na dependência, principalmente, da seleção inicial dos casos. Chapman $^{1}$, ao comentar as dificuldades encontradas no estudo, não de casos individuais, mas de grupos de neuróticos submetidos a determinada terapêutica, chama a atenção para a inconstância deste tipo de paciente em relação ao tratamento e para a necessidade de grande tempo de seguimento $(5,10$ ou 20 anos).

A inconstância dos pacientes é fator que deve ser levado em conta, mas que não pode ser contornado quando a população estudada é a que procura atenção psiquiátrica em lim ambulatório. A observação de pacientes por longo período de tempo poderia dizer melhor das estabilidades das "melhoras" apresentadas, embora, neste caso, as "melhoras" possam não ser, predominantemente, referidas ao tratamento.

A determinação do prognóstico mediante avaliação de "melhoras" na evolução de pacientes, além de "testar" a terapêutica empregada, tem a vantagem de oferecer subsídios para o planejamento de serviços de atenção psiquiátrica de primeira linha.

O propósito deste trabalho é determinar, para uma população de neuróticos em tratamento ambulatorial, a probabilidade de alteração do estado em que se encontram os pacientes (satisfatório ou insatisfatório) no decorrer de consultas sucessivas, durante um período de observação de 12 meses.

\section{MATERIAL E METODOS}

Neste estudo foram incluidos pacientes neuróticos tratados em um ambulatório psiquiátrico. Neste ambulatório são atendidos os funcionários de uma empresa de transportes coletivos de São Paulo e os seus dependentes. Por ocasião da primeira

* Professor Assistente do Departamento de Psicologia Médica e Psiquiatria, Faculdade de Medicina da Organização Santamarense de Educação e Cultura; ** Professora Assistente do Departamento de Medicina Preventiva, Faculdade de Medicina da Universidade de São Paulo.

Nota - Os autores agradecem ao Dr. Jair L. F. Santos pela assessoria no tratamento estatístico dos dados. 
consulta, os funcionários exerciam funções de motorista, cobrador, escriturário, eletricista, mecânico ou fiscal. Os dependentes eram constituídos, na maior parte, pelas esposas e filhos até a idade de 21 anos.

A atençāo psiquiátrica foi dada por um único profissional (um dos autores), que atendeu os casos encaminhados por outros especialistas ou por um médico triagista. O tempo médio de cada consulta foi de 20 minutos. A terapêutica utilizada foi basicamente a medicamentosa, mediante o emprego de ansiolíticos e hipnóticos.

Os pacientes entraram para o estudo em um ponto qualquer do tempo calendário dentro de um periodo de observação de 12 meses (de 1." de agosto de 1972 a 31 de julho de 1973). Neste periodo de tempo foram seguidos 157 pacientes neuróticos, com idade variando entre 20 e 59 anos, os quais constituiram objeto deste estudo.

Foram considerados neuróticos os pacientes cuja capacidade para o trabalho e para a vida social se mostrava perturbada pela angústia ou por seus equivalentes somáticos (sintomas fóbicos, conversivos, obsessivos). Nenhum deles apresentava sintomas como delírios, alucinaçōes, dissociação do pensamento ou distúrbios do humor. Não foram incluidos pacientes disrítmicos, toxicômanos ou com traços delinqüenciais.

$\mathrm{Na}$ primeira consulta todos os pacientes encontravam-se em estado que se convencionou chamar "insatisfatório". Como são dificeis de serem estabelecidos critérios de cura em doenca mental, considerou-se como "melhorados" ou em estado "satisfatório" os pacientes que apresentaram, no decorrer das consultas sucessivas, remissão total ou parcial dos sintomas que motivaram a procura do tratamento.

Para o cálculo da probabilidade de alteração do estado dos pacientes durante o intervalo entre uma dada consulta $x$ e a subseqüente $x+1$, foi utilizado o seguinte procedimento:

Considerando (a) como estado "satisfatório" e (b) como estado "insatisfatório", tem-se:

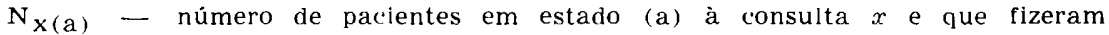

$$
\begin{aligned}
& \text { a consulta } x \text { e a } x+1 \\
& \mathrm{~N}_{\mathbf{X}} \text { (b) - número de pacientes em estado (b) à consulta } x \text { e que fizeram } \\
& \text { a consulta } x \text { e a } x+1
\end{aligned}
$$

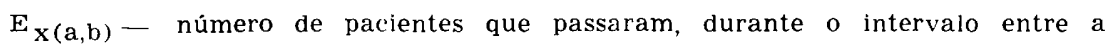

Em cada intervalo entre a consulta $x$ e a $x+1$, pôde-se calcular as probabilidades de "melhora", "piora" ou "inalteração", dado que os pacientes se encontravam em um estado determinado à consulta $x$. Seguem-se as fórmulas utilizadas para o cálculo:

1. Probabilidade de "melhora" da consulta $x$ à $x+1$, dado que os individuos estavam em estado "insatisfatório" à consulta $x$ :

$$
P(S \mid I)=\frac{E_{X(b, a)}}{N_{X(b)}}
$$

2. Probabilidade de "piora" da consulta $x$ à $x+1$, dado que os individuos estavam em estado "satisfatório" à consulta $x$ :

$$
P(I \mid S)=\frac{E_{X(a, b)}}{N_{X(a)}}
$$


3. Probabilidade de inalteração da consulta $x$ à $x+1$, dado que os individuos estavam em estado "satisfatório" à consulta $x$ :

$$
P(S \mid S)=\frac{N_{X}(a)-E_{X}(a, b)}{N_{X(a)}}
$$

4. Probabilidade de inalteração da consulta $x$ à $x+1$, dado que os individuos estavam em estado "insatisfatório" à consulta $x$ :

$$
P(I \mid I)=\frac{N_{X(b)}-E_{X(b, a)}}{N_{X(b)}}
$$

$\mathrm{Na}$ primeira consulta todos os pacientes se encontravam em estado "insatisfatório". Houve certo número que fez a consulta $x$ e nāo a $x+1$. A estes pacientes convencionou-se chamar "perdidos".

Para testar a significancia das diferenças entre as probabilidades observadas de "melhora", "piora" e "inalteração" no decorrer das consultas, utilizou-se procedimento semelhante ao empregado para tábuas de sobrevivências. Neste sentido, os valores de $P(S \mid I), P(I \mid S), P(S \mid S)$ e $P(I \mid I)$ podem ser tratados da mesma forma que os valores $\frac{1_{x+n}}{1}$ numa tábua de sobrevivência ${ }^{5}$.

Exemplifica-se com o teste das diferenças observadas entre os valores de $P(S \mid I)$. Chamando de $P_{I}(S \mid I)$ a probabilidade de "melhora" no intervalo entre a consulta $x_{i}$ e a subsequiente $x_{i}+1$ e de $P_{j}(S \mid I)$ a probabilidade de "melhora" no intervalo entre a consulta $x_{j}$ e a subseqüente $x_{j}+1$, as variâncias de $P_{i}(S \mid I)$ de $P_{j}(S \mid I)$ são dadas por:

$$
\begin{aligned}
& { }_{\sigma_{i}}^{\sigma_{i}}\left\{\mathrm{P}_{\mathrm{i}}(\mathrm{S} \mid \mathrm{I})\right\}=\frac{\left[\mathrm{P}_{\mathrm{i}}(\mathrm{S} \mid \mathrm{I})\right]}{\mathrm{N}_{\mathrm{X}_{\mathrm{i}}(\mathrm{b})}^{\left[1-\mathrm{P}_{\mathrm{i}}(\mathrm{S} \mid \mathrm{I})\right]}} \\
& \sigma_{j}^{2}\left\{P_{J}(S \mid I)\right\}=-\left[P_{j}(S \mid I)\right] \frac{\left[1-P_{J}(S \mid I)\right]}{N_{X_{j}}(b)}
\end{aligned}
$$

E a co-variância entre $P_{i}(S \mid I)$ e $P_{j}(S \mid I)$ é nula ${ }^{3}$. Portanto, para se testar $P_{i}(S \mid I)$ contra $P_{j}(S \mid I)$, usando da aproximação normal, válida para os valores de $P_{1}$ e $P_{j}{ }^{5}$, o teste será o de duas médias, com desvios padrão desconhecidos e não supostamente iguais ${ }^{2}$, como segue:

$$
t_{r}=\frac{P_{j}(S \mid I)-P_{i}(S \mid I)}{\sqrt{\frac{\sigma_{j}}{\sigma_{j}}}+\frac{\sigma_{1}}{N_{X_{j}(b)}}+\frac{N_{X_{i}(b)}}{10}}
$$

Os valores de $\mathrm{t}_{\mathrm{f}}$ têm distribuição $t$ de Student com $f$ graus de liberdade:

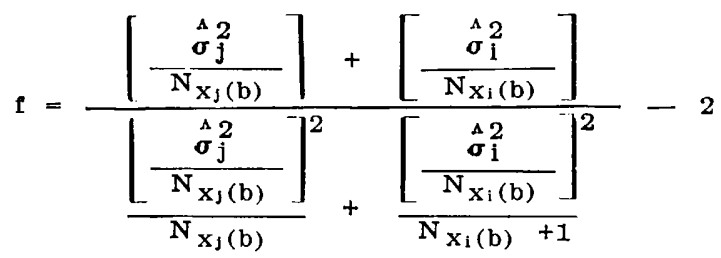

O teste entre os valores observados de $P(I \mid S)$ será feito da mesma maneira. Os valores de $P(I \mid I)$ e de $P(S \mid S)$ são complementares aos de $P(S \mid I)$ e de $P(I \mid S)$, respectivamente. 


\section{RESULTADOS E COMENTARIOS}

O prognóstico de uma doença derivado, não de casos descobertos e seguidos na população geral, mas de casos que se apresentam para tratamento, pode produzir um quadro diferente do real. Contudo, a dificuldade de realização de inquéritos populacionais e de seguimento de grande número de pessoas, justifica a utilização de pacientes que se apresentam para tratamento médico. Os dados resultantes deste tipo de estudo são úteis para o conhecimento da evolução de pacientes submetidos a determinada terapêutica, dando assim elementos para o planejamento de atenção psiquiátrica ao nivel do ambulatório. A avaliação da eficiência de uma modalidade terapêutica, oferecida a um grupo de pacientes, fornece subsídios para possíveis reformulações e para a previsão de recursos necessários neste tipo de prestação de serviços.

O prognóstico seria melhor determinado se se levasse em consideração fatores que, sabidamente, pudessem influir sobre ele, evidenciados em estudos como o de Gunderson \& Arthur ${ }^{4}$. No presente estudo, a estratificação do grupo segundo as variáveis mais importantes resultaria em um número pequeno de pacientes em cada classe, invalidando qualquer conclusão. Para atenuar a influência do fator idade, só foram considerados pacientes adultos de 20 a 59 anos. O grupo era homogêneo quanto ao nivel sócio-econômico. A distribuição segundo idade e sexo è apresentada na tabela 1.

\begin{tabular}{|c|c|c|c|c|c|c|c|c|c|}
\hline \multirow[t]{2}{*}{ Sexo } & \multicolumn{7}{|c|}{ Idade * } & \multirow[t]{2}{*}{ Idade mediana * } & \multirow[t]{2}{*}{ Total } \\
\hline & 20 & $1-30$ & 30 & $1-40$ & 40 & $1-50$ & $501-60$ & & \\
\hline $\mathbf{M}$ & & 34 & & 20 & & 21 & 7 & 33 & 82 \\
\hline$F^{*}$ & & 23 & & 27 & & 24 & 1 & 35 & 75 \\
\hline Total & & 57 & & 47 & & 45 & 8 & 34 & 157 \\
\hline
\end{tabular}

Os intervalos medianos entre as consultas foram de 31 dias entre $1^{*}$ e $2^{a}, 31,5$ dias entre $2 .^{a}$ e $3 .^{a}, 42$ dias entre $3 .^{a}$ e $4^{a}$ e 32,5 dias entre $4^{a}$ e $5 .^{\text {a }}$. As probabilidades de "melhora", "piora" e "inalteração", bem como o número e a proporção de "perdidos" são apresentados na tabela 2. Não foram calculadas as probabilidades a partir da $5 .^{\mathrm{a}}$ consulta devido ao pequeno número de pacientes.

Observa-se, na tabela 2, que a probabilidade de "melhora" aumenta de uma consulta para outra, alcançando o seu ma:or valor entre $4 .^{\mathrm{a}}$ e $5 .^{\text {a }}$ consultas $(83,3 \%)$. Com a probabilidade de "piora" ocorre o inverso (entre 4." e $5 .^{u}$ consultas foi de $30 \%$ ). A probabiliaade de inalteração uma vez que o indivíduo esteja em estado "satisfatório" também é máxima entre 4." e 5." consultas $(70 \%)$. A probabilidade de inalteração no estado "insatisfatório" é mínima entre $4 .^{\mathrm{a}}$ e $5 .^{\mathrm{a}}$ consultas $(16,7 \%)$. As diferenças observadas entre 


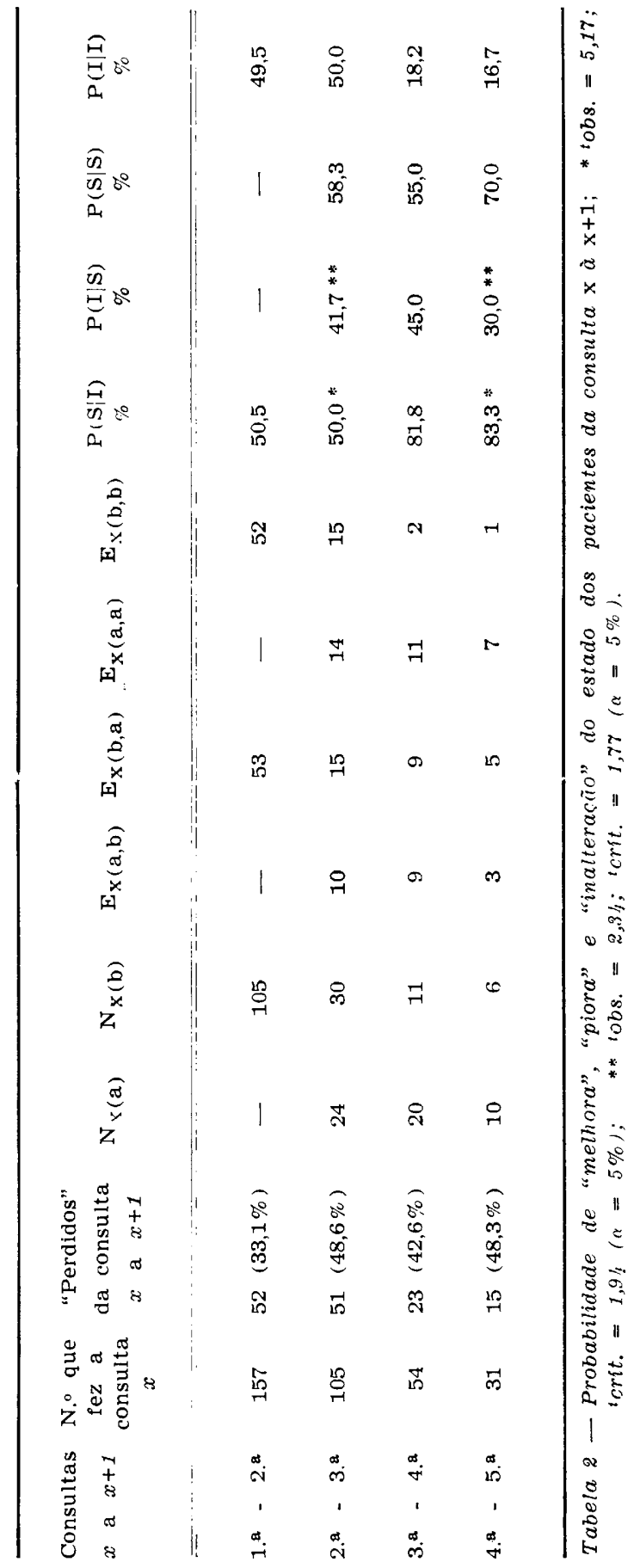


$2^{a}-3^{a}$ e $4 .^{a}-5 .^{a}$ consultas são significantes ao nível de $5 \%$. A diferença observada para probabilidade de "piora" entre $2 .^{\mathrm{a}}-3^{\mathrm{a}}$ (41,7\%) e $3^{\mathrm{q}}-4^{\mathrm{a}}$ consultas (45\%) não é significante ao nível de $5 \%$.

Dos pacientes considerados "perdidos" de observação, apenas 3 o foram porque tiveram alta médica. As proporções de "perdidos" são relativamente altas, expressando a inconstância do paciente neurótico no tratamento, comentada por Chapman ${ }^{1}$. Considerando que a maioria destes pacientes não tem acesso a outro serviço de assistência médica e que a eles foi dada a oportunidade de continuar o tratamento, é de se supor que não tenham voltado por terem apresentado "melhora". Esta hipótese é apoiada pelo retorno de vários pacientes muito tempo após a data marcada referindo estarem assintomáticos naquele intervalo. É possível, portanto, que se estes pacientes tivessem sido seguidos durante todo o período, a probabilidade de "melhora" fosse maior que a observada.

\section{RESUMO}

Procurou-se determinar o prognóstico de pacientes neuróticos em tratamento ambulatorial mediante o cálculo das probabilidades de "melhora", "piora" e "inalteração" em consultas sucessivas. Foi, assim, analisada a evolução de 157 pacientes de 20 a 59 anos que entraram para o estudo em um ponto qualquer durante um periodo de observação de 12 meses.

\section{SUMMARY}

\section{Prognosis of neurotic patients on outpatient care}

The prognosis of neurotic patients followed on a outpatient service was determined by calculation of probabilities of "improvement", "worsening" or "lack of changes" on successive examinations. These criteria were established for evaluation of 157 patients, 20 to 59 years old, who entered the study during a 12 months observation period.

\section{REFERENCIAS}

1. CHAPMAN, A. H. - The problem of prognosis in psychoneurotic illness. Amer. J. Psychiat. 119:768, 1963.

2. DIXON, W. J. \& MASSEY Jr., F. J. - Introducción al Análisis Estadístico. Versão castelhana da 2.a ed. Ediciones Castilha, Madrid, 1966.

3. FENICHEL, O. - Teoría Psicoanalitica de las Neurosis. Versão castelhana da 3. ${ }^{\text {a }}$ ed. Ed. Paidós, Buenos Aires, 1966.

4. GUNDERSON, E. K. E. \& ARTHUR, R. J. - Prognostic indicators in psychosis and neurosis. J. Abnorm. Psychol. 73:468, 1968.

5. SANTOS, J. L. F. - Contribuição para o tratamento estocástico da tábua de sobrevivência e suas aplicações. São Paulo (Tese de doutoramento — FSPUSP), 1972.

Departamento de Medicina Preventiva - Faculdade de Medicina da Universilade de São Paulo - Av. Dr. Arnaldo 455 - 01248 São Paulo, SP - Brasil. 